\title{
STUDY ON THE CORRELATION BETWEEN TAPSE, SA AND RV FAC IN PATIENTS WITH ACUTE MYOCARDIAL INFARCTION
}

\author{
Karodi Murali Krishna ${ }^{1}$
}

${ }_{1}^{1}$ Assistant Professor, Department of Cardiology, Guntur Medical College, Guntur.

\begin{tabular}{l}
\hline ABSTRACT \\
BACKGROUND \\
Several echocardiographic methods have been proposed to rapidly and routinely analyse Right Ventricular (RV) performance in \\
various clinical settings. Among these, TAPSE, Sa and RV FAC are simple, rapid and feasible methods which were correlated with the \\
standard methods of RV functional evaluation.
\end{tabular}

\section{MATERIALS AND METHODS}

Current study evaluated right ventricular function by echocardiography in 200 consecutive patients of acute ST elevation myocardial infarction admitted to Osmania General Hospital, Hyderabad during early hospitalisation period. We assessed right ventricular function by TAPSE, Sa and RV FAC.

\section{RESULTS}

There was significant and high correlation between all the three parameters (TAPSE, Sa and RV FAC) used for assessing right ventricular function. The correlation between TAPSE and Tricuspid annular peak systolic velocity (Sa) was highest compared to other parameter correlations. This is probably because both the parameters are measures of longitudinal contraction of right ventricle.

\section{CONCLUSION}

There is high correlation between TAPSE, Sa and RV FAC in assessing RV function after acute MI.

\section{KEYWORDS}

Correlation, Acute Myocardial Infarction, TAPSE, Sa, RV FAC.

HOW TO CITE THIS ARTICLE: Krishna KM. Study on the correlation between TAPSE, Sa and RV FAC in patients with acute myocardial infarction. J. Evolution Med. Dent. Sci. 2017;6(95):6923-6926, DOI: 10.14260/jemds/2017/1502

\section{BACKGROUND}

In 1616, Sir William Harvey was the first to describe the importance of right ventricular (RV) function in his seminal treatise, De Motu Cordis: "Thus the right ventricle may be said to be made for the sake of transmitting blood through the lungs, not for nourishing them".[1]

The assessment of right ventricular function has become increasingly important in management of various cardiovascular diseases including acute myocardial infarction. Evaluation of right ventricular systolic function is very important for its clinical and prognostic value. The accurate non-invasive assessment of right ventricular systolic function is, however, difficult due to its complex structure, geometry and anatomy of right ventricle. ${ }^{[2]}$

The accurate non-invasive methods for evaluation of RV systolic function such as radionuclide ventriculography and Magnetic resonance imaging are relatively expensive, time consuming and cumbersome and are not feasible for the evaluation of patients with acute myocardial infarction who are acutely ill and unstable.[3]

Several echocardiographic methods have been proposed to rapidly and routinely analyse RV performance in various

'Financial or Other Competing Interest': None.

Submission 31-10-2017, Peer Review 04-12-2017,

Acceptance 11-12-2017, Published 23-12-2017.

Corresponding Author:

Dr. K. Murali Krishna

D.no. 4-19-115/1,

Vikar Nagar, 4th Line,

Guntur, Andhra Pradesh- 522006, India.

E-mail: drmuralikrishnakarodi@gmail.com

DOI: $10.14260 /$ jemds $/ 2017 / 1502$ clinical settings. Among these, Tricuspid Annular Plane Systolic Excursion (TAPSE), Doppler tissue imaging for assessment of peak systolic velocity of tricuspid lateral annulus (Sa) and Right Ventricular Fractional Area Change (RV FAC) are simple, rapid and feasible methods which were correlated with the standard methods of RV functional evaluation. Various studies demonstrated that right ventricular involvement in acute myocardial infarction was associated with poor prognosis. Decreased Right ventricular systolic function is a major risk factor for sudden cardiac death and heart failure. ${ }^{[3,4]}$

\section{MATERIALS AND METHODS}

Study population: 200 consecutive patients admitted with acute ST Elevation Myocardial Infarction to the ICCU of Osmania General Hospital, Hyderabad during the period of January 2013 to December 2013.

Study design: Hospital-based descriptive study

\section{Inclusion Criteria}

All patients who presented with ST Elevation Myocardial Infarction for the first time with in the 5 days of onset of symptoms, irrespective of age, gender and treatment status.

\section{Exclusion Criteria}

Patients with past history of Q-wave myocardial Infarction, Chronic obstructive pulmonary disease, Pulmonary arterial hypertension, Chronic Rheumatic heart disease, Cardiomyopathies. Congenital heart diseases, Poor echo window for assessment of Right Ventricle and those unwilling for the study. 
All patents were clinically evaluated. Detailed history noted from patients, attendants and from first clinical clerking from the documents available. Detailed general examination done. Vitals recorded. JVP evaluated, a thorough cardiovascular examination done. All the details entered in a proforma.

ECGs recorded at admission with BPL Cardiart machine, a 12-lead single channel ECG, with additional leads of V3R, V4R in case of inferior wall MI, at $25 \mathrm{~mm} /$ second speed and at 10 $\mathrm{mm} / \mathrm{mV}$ standardisation. All the patients were subjected to two-dimensional echocardiography, M-mode and Doppler echocardiography within one day of admission. Echocardiographic examinations were performed on PHILIPS iE 33 machine with a system equipped with Doppler tissue imaging.

\section{Assessment of Right Ventricular Function}

This was done by the following methods:

1. Tricuspid Annular Plane Systolic Excursion (TAPSE).

2. Right ventricular Fractional Area Change (RV FAC).

3. Doppler tissue imaging for assessment of peak systolic velocity of tricuspid lateral annulus. (Sa).

\section{Statistical Analysis}

Data was entered and analysed using SPSS 16.0 statistical package. All categorical variables were expressed as numbers and percentages. Pearson's correlation was used to determine significant correlations between the variables and scatter plot to depict the correlations. A value of $p<0.05$ was considered statistically significant.

\section{RESULTS}

Current study evaluated right ventricular function by echocardiography in 200 consecutive patients of acute ST elevation myocardial infarction admitted to Osmania General Hospital, Hyderabad during early hospitalisation period. We assessed right ventricular function by TAPSE, Sa and RV FAC.

\section{Correlation between TAPSE, Sa and RV FAC: \\ Correlation between TAPSE and Sa}

The relation between TAPSE and Tricuspid lateral annular peak systolic velocity by tissue Doppler (Sa) was shown in the figure below. TAPSE correlated significantly with Sa. Pearson's correlation coefficient $(r)=0.719$, $p$-value $=<0.001$.

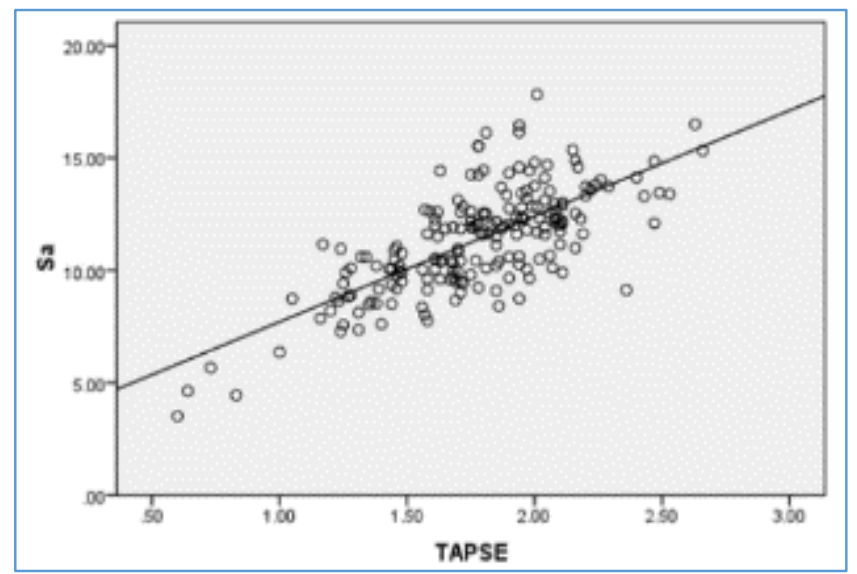

Figure 1. Scatter Plot showing Correlation between Sa and TAPSE
Correlation between TAPSE and RV FAC

The relation between TAPSE and RV FAC were depicted in figure below. TAPSE correlated significantly with RV FAC with Pearson's correlation coefficient: 0.435 , p-value $=<0.001$

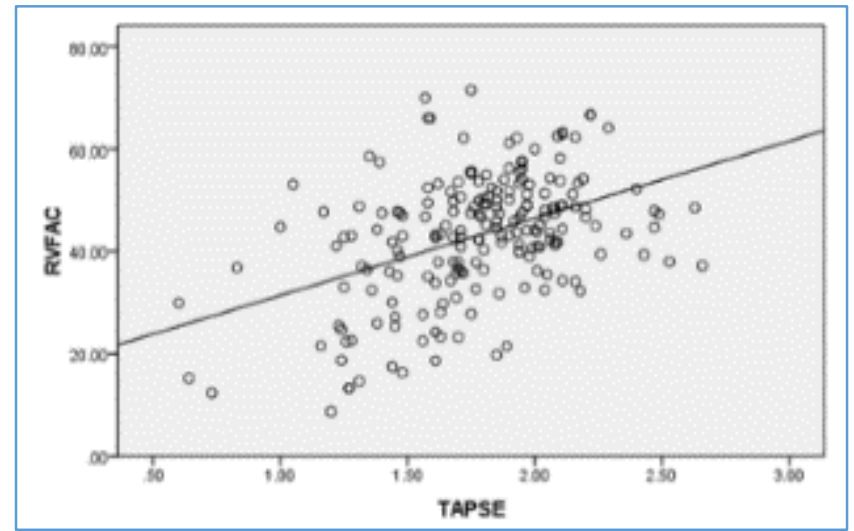

Figure 2. Scatter Plot showing Correlation between RV FAC and TAPSE

\section{Correlation between Sa and RV FAC}

Correlation between tricuspid annular peak systolic velocity (Sa) and RV FAC; Pearson's Correlation Coefficient ( $r$ ) $=0.445$, $\mathrm{P}<0.001 . \mathrm{N}=200$.

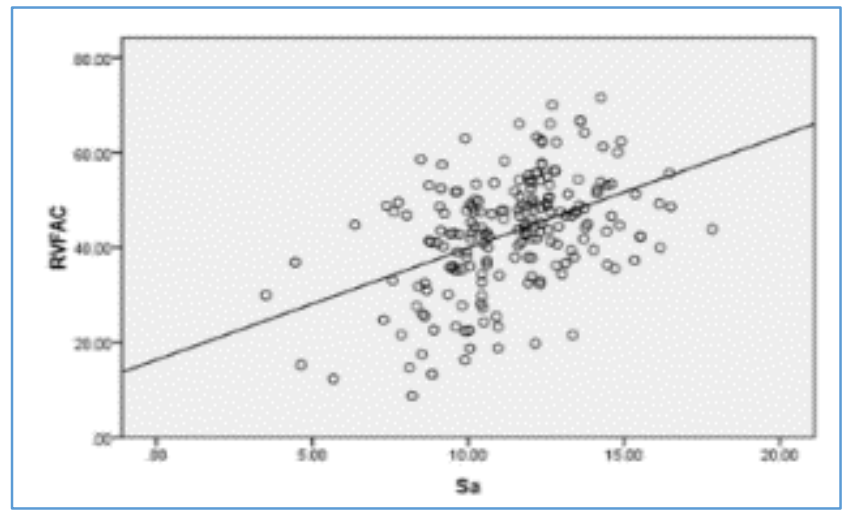

Figure 3. Scatter Plot showing Correlation between RV FAC and Sa

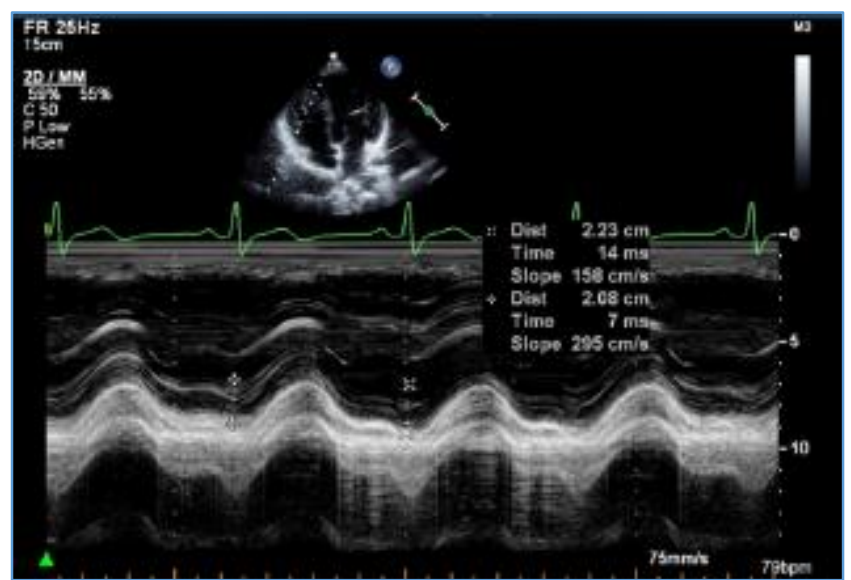

Figure 4. Measurement of Tricuspid Annular Plane Systolic Excursion (TAPSE) by M-mode Echocardiography 


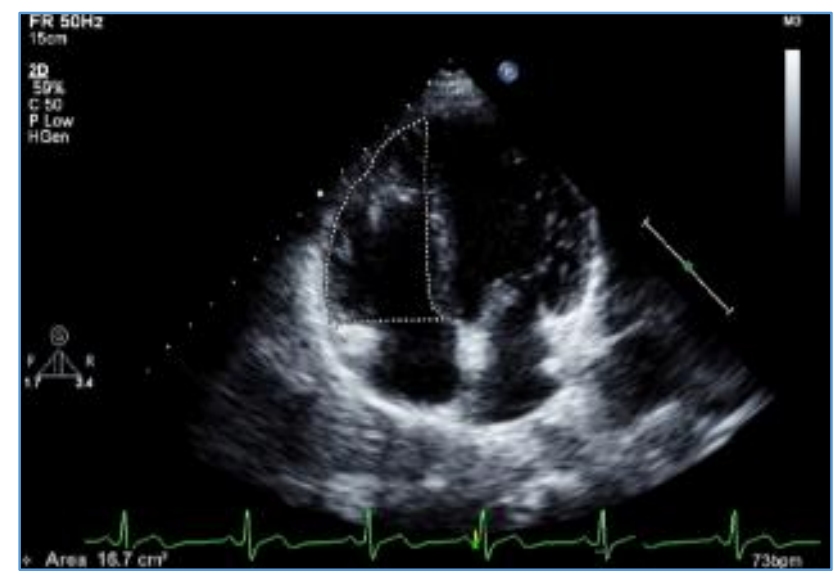

Figure 5. Measurement of RV End diastolic Area in Apical Four-chamber View for Calculating RV Fractional Area Change

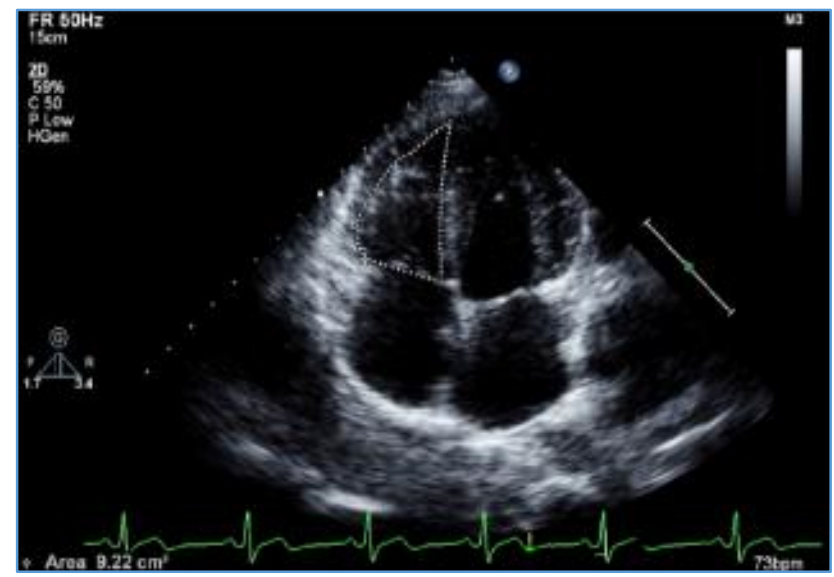

Figure 6. Measurement of RV End systolic Area in Apical Four-chamber View for Calculating RV Fractional Area Change

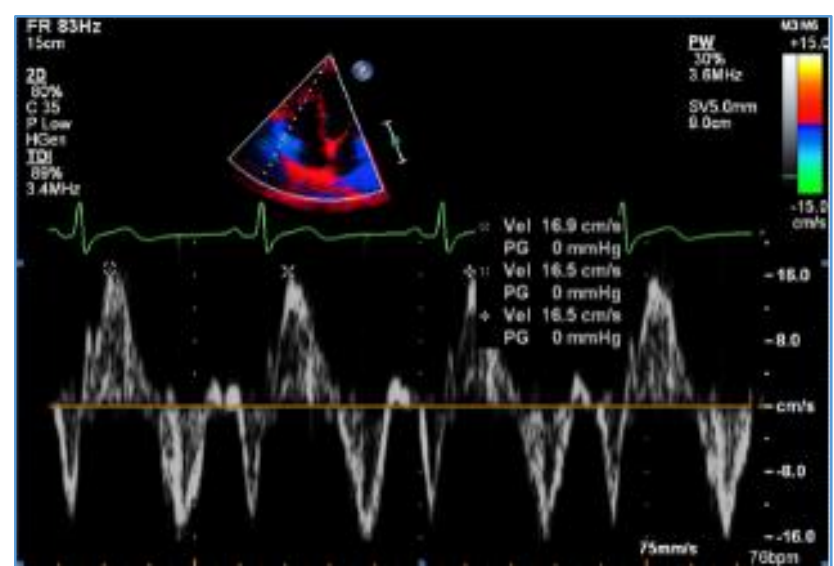

Figure 7. Tissue Doppler Imaging showing Measurement of Tricuspid Annular Peak Systolic Velocity (Sa)

\section{DISCUSSION}

There was significant and high correlation between all the three parameters (TAPSE, Sa and RV FAC) used for assessing right ventricular function. The correlation between TAPSE and Tricuspid annular peak systolic velocity ( $\mathrm{Sa}$ ) was highest compared to other parameter correlations. This is probably because both the parameters are measures of longitudinal contraction of right ventricle.

However, on extensive review of literature, there was no study which correlated TAPSE, Sa and RV FAC. In a study by
Kaul et al,[5] TAPSE correlated well with RV ejection fraction by radionuclide ventriculography, they proposed a cut-off value of TAPSE $<1.5 \mathrm{~cm}$ to differentiate patients with RV dysfunction to those with normal RV function. In another study by Meluzin et al,[6] there was good correlation between systolic tricuspid annular velocity and RV EF by radionuclide ventriculography and they proposed $\mathrm{Sa}<11.5 \mathrm{~cm} / \mathrm{s}$ predicted RV dysfunction with a sensitivity of $90 \%$ and specificity of $85 \%$. In another study, RV FAC correlated well with RV EF by MRI,[7] and Weyman ${ }^{[8,9]}$ proposed a cut-off value of $<32 \%$ to differentiate RV dysfunction and normal RV function. But the cut-off values proposed above show considerable variability in predicting RV dysfunction in our study.

In our study, we correlated the assessment of RV function by three different echocardiographic parameters and found that all the three parameters namely TAPSE, Sa and RV FAC are significantly correlated. Although all the three parameters have high and significant correlation there was considerable variability in predicting RV dysfunction. As we did not compare with gold standard parameters like Radionuclide ventriculography or MRI derived RVEF, there is a need for a study which correlates all the three parameters with gold standard RV functional parameters to predict the interchangeable cut-off value for all the three echocardiographic parameters.

\section{CONCLUSION}

Nearly one fourth of patients with acute ST elevation myocardial infarction had right ventricular dysfunction. Patients with RV dysfunction had significantly large LV infarct size and low LV ejection fraction. Patients with RV dysfunction were significantly older and have significantly higher hospital mortality after acute myocardial infarction. There is high correlation between TAPSE, Sa and RV FAC in assessing RV function after acute MI.

\section{REFERENCES}

[1] Chin KM, Kim NH, Rubin LJ. The right ventricle in pulmonary hypertension. Coron Artery Dis 2005;16(1):13-8.

[2] Haddad F, Hunt SA, Rosenthal DN, et al. Right ventricular function in cardiovascular disease, part I: anatomy, physiology, aging, and functional assessment of the right ventricle. Circulation 2008;117(11):143648.

[3] Jurcut R, Giusca S, La Gerche A, et al. The echocardiographic assessment of the right ventricle: what to do in 2010? Eur J Echocardiogr 2010;11(2):8196.

[4] Jiang L. Right ventricle. In: Weyman AE, (eds). Principles and practice of echocardiography. Lippincott Williams and Wilkins; Baltimore, 1994:901-21.

[5] Kaul S, Tei C, Hopkins JM, et al. Assessment of right ventricular function using two-dimensional echocardiography. Am Heart J 1984;107(3):526-31.

[6] Vogel M, Schmidt MR, Kristiansen SB, et al. Validation of myocardial acceleration during isovolumic contraction as a novel non-invasive index of right ventricular contractility: comparison with ventricular pressurevolume relations in an animal model. Circulation 2002;105(14):1693-9. 
[7] Anavekar NS, Gerson D, Skali H, et al. Two-dimensional assessment of right ventricular function: an echocardiographic-MRI correlative study. Echocardiography 2007;24(5):452-6.

[8] Lang RM, Bierig $M$, Devereux RB, et al. Recommendations for chamber quantification: a report from the American Society of Echocardiography's guidelines and standards committee and the chamber quantification writing group, developed in conjunction with the European association of echocardiography, a branch of the European society of cardiology. J Am Soc Echocardiogr 2005;18(12):1440-63.

[9] Weyman A. Practices and principles of echocardiography. 2nd edn. Philadelphia: Lippincott, Williams and Wilkins, 1994. 